\title{
Physical activity associates with enhanced immunogenicity of an inactivated virus vaccine against SARS-CoV-2 in patients with autoimmune rheumatic diseases
}

\author{
Bruno Gualano ( $\nabla$ gualano@usp.br) \\ University of Sao Paulo \\ Italo Lemes \\ University of Sao Paulo https://orcid.org/0000-0001-9245-287X \\ Rafael Silva \\ University of Sao Paulo
}

Ana Pinto

University of Colorado

Bruna Mazzolani

University of Sao Paulo

Fabiana Smaira

University of Sao Paulo

Sofia Sieczkowska

University of Sao Paulo

\section{Nadia Aikawa}

Hospital das Clinicas, University of Sao Paulo

\section{Sandra Pasoto}

Hospital das Clinicas, University of Sao Paulo

\section{Ana Medeiros-Ribeiro}

Hospital das Clinicas, University of Sao Paulo

\section{Carla Saad}

Hospital das Clinicas, University of Sao Paulo

\section{Emily Yuk}

Hospital das Clinicas, University of Sao Paulo

Clovis Silva

Hospital das Clinicas, University of Sao Paulo

Paul Swinton

Robert Gordon University

\section{Pedro Hallal}


Hamilton Roschel

University of Sao Paulo

Eloisa Bonfa

Hospital das Clinicas, University of Sao Paulo

\section{Article}

Keywords:

Posted Date: August 9th, 2021

DOl: https://doi.org/10.21203/rs.3.rs-782398/v1

License: (c) (1) This work is licensed under a Creative Commons Attribution 4.0 International License. Read Full License 


\section{Abstract}

Immunocompromised individuals show lower vaccine immunogenicity, which may be modulated by physical activity. This prospective cohort study within a phase-4 vaccination trial investigated whether physical activity is associated with enhanced immunogenicity of Coronavac (SARS-CoV-2 inactivated vaccine) in patients with autoimmune rheumatic diseases (ARD) $(n=898)$ and non-ARD $(n=197)$ individuals without pre-existing immunogenicity to SARS-CoV-2 after receiving a two-dose vaccine schedule. Seroconversion rates of total anti-SARS-CoV-2 S1/S2 IgG (SC), geometric mean titers of antiS1/S2 IgG (GMT), factor-increase in GMT (FI-GMT), frequency of neutralizing antibody (NAb), and median neutralizing activity were assessed. After controlling for covariates, active patients $(\geq 150$ min/week) exhibited greater SC (OR: 1.4 [95\%Cl: 1.1-2.0]), GMT (32\% [95\%Cl: 8.8-60) and FI-GMT (33\% [95\%Cl: 9.6-63\%]) vs. inactive. Cluster analysis (physical activity/sedentary status) revealed greater GMT (43.0\% [95\% Cl: 11.0-84.0\%) and FI-GMT (48.0\% [95\% Cl: 14.0-92.0\%]) in active/non-sedentary ( $\geq 150$ $\mathrm{min} / \mathrm{week} /<8 \mathrm{~h} /$ day) vs. inactive/sedentary (<150 min/week/>8h/day) ARD. A dose-response was observed, with greater benefits for $\geq 350 \mathrm{~min} /$ week of physical activity (OR: 1.6 [95\%Cl: 1.1-2.4]; 41\% [95\% Cl: 10-80\%]; 35\% [95\% Cl: 4.3-74], for SC, GMT, and FI-GMT, respectively). Greater SC (OR: 9.9 [95\%Cl: 1.1-89.0]) and GMT (26\% [95\%Cl: 2.2-56.0\%]) were observed in active vs. inactive non-ARD. A physically active lifestyle may enhance SARS-CoV-2 vaccine immunogenicity, a finding of particular clinical relevance for immunocompromised individuals.

\section{Introduction}

Vaccines have played a vital role in controlling the COVID-19 pandemic, as observed in countries welladvanced in rolling out vaccination. ${ }^{1-3}$ However, a concern remains that vaccine-induced immunogenicity might not be as high in immunocompromised individuals, such as those with autoimmune rheumatic diseases (ARD), neoplasia, transplant recipients and patients with HIV.

In fact, literature has been controversial regarding the SARS-CoV-2 vaccine responses in these groups. In a small study involving patients with chronic inflammatory diseases $(n=26)$, all patients developed antibody responses after SARS-CoV-2 mRNA vaccination, but they exhibited reduced IgG and neutralizing antibodies levels compared to healthy controls. ${ }^{4}$ In addition, a reduced anti-spike antibody response was showed after the $1^{\text {st }}(17 \%)$ and $2^{\text {nd }}$ doses $(54 \%)$ of SARS-CoV-2 mRNA 1273 or BNT162b2 vaccine in solid organ transplant recipients. ${ }^{5,6}$ In a retrospective cohort study of patients with a variety of immunemediated inflammatory diseases including ARD $(n=84), 91 \%$ produced detectable neutralizing activity to BNT162b2 mRNA SARS-CoV-2 vaccine. ${ }^{7}$ Furthermore, a non-controlled, prospective cohort study with ARD patients ( $n=123)$ showed presence of anti-receptor-binding domain (RBD) antibodies in $74 \%$ of them, but lower IgG and neutralizing antibody levels compared to healthy controls ${ }^{8}$. In line with this finding, we recently showed that an inactivated virus vaccine against SARS-CoV-2 (CoronaVac) - which accounts for $\sim 45 \%$ of the administered vaccines in Brazil - elicited a lower but still clinically effective response in a large cohort of natients with ARD ( $n=910)$ compared to controls. ${ }^{9}$ Although the impact of this reduced Loading [MathJax]/jax/output/CommonHTML/jax.js 
immunogenicity upon vaccine effectiveness remains unknown, efforts to determine modifiable factors potentially able to enhance vaccine response are of utmost importance, particularly in immunocompromised individuals.

There has been a long-stand notion that regular physical activity may enhance immune system response. Exercise has been shown to reduce chronic low-grade inflammation and has been linked to increased Tcell proliferation and cytokine production following antigenic stimulation, increased neutrophil phagocytic activity, and increased natural killer cell cytolytic activity. ${ }^{10}$ There is also evidence that physical activity can improve immune responses to influenza and pneumococcal vaccines, hastening the recovery following experimental rhinovirus infection. ${ }^{10} \mathrm{~A}$ recent meta-analysis from 6 studies involving 497 individuals vaccinated against H1N1, H3N2, influenza type-B, pneumococcal and varicella zoster virus showed that pooled antibody concentration after vaccination is higher with an adjunct physical activity program, leading to the speculation that physical activity may "strengthen the potency of immunization programs and help mitigate the impact of pandemics such as the COVID-19". ${ }^{11}$

To our knowledge, this is the first study to investigate the influence of physical activity on the immunogenicity of a vaccine against SARS-CoV-2 (CoronaVac) in a large cohort of patients with ARD. As a secondary objective, we also assessed whether physical activity status affects immunogenicity in nonARD individuals. Our working hypothesis was that physically active ARD patients would experience better vaccine-induced immune responses compared to their inactive peers.

\section{Results}

\section{Participants}

A total of 1418 ARD patients were recruited, and 225 were excluded for the following reasons: 24 acute febrile illness/symptoms compatible to COVID-19 at vaccination day or real-time RT-PCR confirmed COVID-19 less than four weeks before vaccination day, 1 demyelinating disease, 25 previous vaccination with any SARS-Cov-2 vaccine, 1 inactivated virus vaccination, 161 individuals did not accept to participate in the study, and 13 hospitalized patients. Subsequently, 542 controls were recruited, but 50 refused to participate. The remaining 1193 ARD patients and 492 non-ARD individuals received the $1^{\text {st }}$ dose, but 232 (19.4\%) ARD patients and 191 (38.8\%) non-ARD individuals had positive baseline IgG serology and/or NAb and were excluded. Also, 63 ARD (5.3\%) patients and 104 non-ARD individuals (21.1\%) did not respond to the physical activity survey and were excluded (Supplementary Figure 2). The remaining ARD patients ( $n=898$; Table 1$)$ and non-ARD individuals ( $n=197$; Supplementary Table 1) were analyzed.

Physically active ARD patients $(\mathrm{n}=494)$ were significantly younger $(P<.001)$, and more frequently used prednisone $(P<.001)$ and biologic $(P<.001)$ than inactive $(\mathrm{n}=404)$. Active $(\mathrm{n}=128)$ and inactive $(\mathrm{n}=69)$ nonARD individuals did not statistically differ in age, sex and BMI ( $P=.397)$ (Table 2). 


\section{Unadjusted analysis}

Figure 1 presents immunogenicity data for active vs. inactive ARD patients and non-ARD individuals. After vaccination, frequency of SC $(P<.001)$, GMT $(P<.001)$, FI-GMT $(P<.001)$, frequency of NAb $(P=.022)$ and its neutralizing activity $(P<.001)$ were greater in active vs. inactive ARD patients. Active non-ARD individuals exhibited greater SC than inactive ones $(P=.038)$.

\section{Adjusted analysis}

Figure 2 presents the regression models controlling for covariates in ARD patients. In general, older age, $\mathrm{BMl}>30 \mathrm{~kg} / \mathrm{m}^{2}$, and use of prednisone, biologics and immunosuppressants were the factors more strongly associated with poor immunogenicity, while being physically active was associated with better immunogenicity.

Point estimates from logistic regression models indicated greater odds of SC in physically active vs. inactive patients (OR: 1.4 [95\% Cl: 1.1 to 2.0]). ARD patients who were physically active also exhibited approximately $30 \%$ greater GMT (32\% [95\% Cl: 8.8 to 60$]$ ) and FI-GMT (33\% [95\% Cl: 9.6 to $63 \%])$ than inactive ones. The associations between physical activity and neutralizing activity $(4.5 \%$ [95\% Cl: -0.1 to 9.1\%]) and neutralizing antibodies (OR: 1.2 [95\% Cl: 0.9 to 1.6]) were non-significant.

Cluster exploratory analysis of physical activity/sedentary status revealed significantly greater percent changes for GMT (43.0\% [95\% Cl: 11.0 to $84.0 \%$ ]) and FI-GMT (48.0\% [95\%Cl: 14.0 to $92.0 \%]$ ) in active/non-sedentary vs. inactive/sedentary ARD patients. Importantly, active/sedentary showed no difference in GMT and FI-GMT compared with inactive/sedentary, suggesting that sedentary behavior may have overridden the influence of physical activity (Figure 3). The other exploratory analysis showed a dose-response between physical activity volumes and SC, GMT and FI-GMT, with the greatest benefits seen for $\geq 350 \mathrm{~min} /$ week of physical activity (OR: 1.6 [95\%Cl: 1.1 to 2.4 ], $41 \%$ [95\% Cl: 10 to $80 \%$ ] and 35\% [95\% Cl: 4.3 to 74] for SC, GMT and FI-GMT) (Supplementary Figure 3).

Among non-ARD, point estimates from logistic regression models indicated greater odds of SC with a wide $\mathrm{Cl}$ range in active vs. inactive individuals (OR: 9.9 [95\% Cl: 1.1 to 89.0$]$ ). Active individuals showed $26.0 \%$ greater GMT (95\% Cl: 2.2 to $56.0 \%$ ) and $24.0 \%$ FI-GMT (95\% Cl: -9.4 to $71.0 \%$ ) compared to inactive, although Cls overlapped 1 for FI-GMT. Frequency of NAb positivity and neutralizing activity did not significantly differ between active and inactive individuals (Figure 4).

\section{Discussion}

To our knowledge, this is the first evidence that being physically active may enhance immunogenicity of a vaccine against SARS-CoV-2 in a large cohort of patients with ARD. Additionally, a similar benefit was Loading [MathJax]/jax/output/CommonHTML/jax.js 
observed in a small cohort of non-ARD individuals. This finding suggests that physical activity may boost vaccine response, which is particularly relevant to immunocompromised individuals who are prone to diminished immunogenicity.

Vaccination is a major strategy in reducing mortality and morbidity rates for several infectious diseases, ${ }^{12}$ including COVID-19. ${ }^{13}$ In countries with high capacity of vaccine acquisition and rapid rollouts, both new cases and deaths have been dramatically reduced. However, vaccine efficacy varies between individuals, with particularly low responses found in those with reduced immune function. ${ }^{14,15}$ mRNA vaccines against SARS-CoV-2 can elicit a reduced humoral response in older individuals and in ARD patients, ${ }^{4-6,16}$ a finding recently extended to CoronaVac, ${ }^{9}$ which has been largely used in highly populated countries, and recently approved for emergency use by WHO ${ }^{17}$ Indeed, previous data from this trial point out to lower SC (70.4 vs. $95.5 \%)$ and titers (12.1 vs. 29.7), frequency of NAb positivity (56.3 vs. $79.3 \%$ ) and neutralization activity (58.7 vs. $64.5 \%$ ) in ARD patients vs. controls. ${ }^{9}$ It becomes clear that the search for adjuvants to enhance vaccine response and improve protection from disease infection is of great clinical importance. Chief amongst these is physical activity, which has been deemed as a behavioral intervention able to boost immune function in different scenarios, thereby potentially serving as an adjuvant to improve vaccine response, including that against SARS-CoV-2. This hypothesis was tested in the present study.

Both observational and interventional studies have shown that habitually physically active individuals, or those receiving exercise interventions, present with higher concentration of IgG and IgM following influenza and keyhole limpet haemocyanin (KLH) vaccination. ${ }^{18-25}$ Apart from studies involving older individuals, evidence that physical activity may confer better vaccine responses in those with less functional immunity is lacking. In this regard, our data bring novel evidence that, compared to their inactive counterparts, physically active ARD patients may have higher SC rates, GMT and FI-GMT and a trend to higher neutralizing activity, even after controlling for several covariates, including age, sex, BMI and medications. Of relevance, the positive association of physical activity with GMT (+32\%) was diametrically opposite to those of age $(-33 \%)$, obesity $(-30 \%)$ and medications (-27 to $-48 \%)$, which underscores the potential importance of a physically active lifestyle in counteracting factors known to impair immunogenicity. Furthermore, our exploratory analysis suggests that the benefits of being physically active (i.e., meeting the minimum recommended amount of physical activity) on vaccine immunogenicity tends to wane owing to sedentary behavior (i.e., too much sitting), a finding that has been observed in population-based studies for all-cause mortality, ${ }^{26,27}$ and that requires confirmation for vaccines responses. We also observed a direct dose-response relationship between physical activity volume and SC, GMT, and FI-GMT. Although current evidence does not yet provide specific information about how intensity, frequency, duration and type of physical activity influence vaccine responses, ${ }^{11}$ the present findings suggest that engaging in at least $150 \mathrm{~min} /$ week of moderate-to-vigorous physical activity while avoiding excessive sitting time may enhance immunogenicity to vaccination against SARSCoV-2, with higher physical activity amounts ( $\geq 350 \mathrm{~min} /$ week) possibly offering greater benefits. 
Hypothetically, young healthy adults might be less responsive to the benefits of physical activity on immunogenicity, since the robust response to most vaccinations in this population may mask more subtle effects of exercise, whereas in those with weaker immune function and higher variability, the immunoenhancement effects may be more noticeable. ${ }^{13}$ Similar to ARD patients, however, we observed a positive association between physical activity and SC rates and GMT in non-ARD individuals. This suggests the potential applicability of our findings in a more generalized context; nonetheless, these should be validated in a larger cohort of non-immunosuppressed individuals.

The mechanisms by which regular physical activity enhance vaccination responses are not fully understood. However, it is known that moderate-to-vigorous physical activity is able to improve immune function, which is reflected in greater antibody or cell-mediated responses to vaccination. ${ }^{13,28}$ Even a single bout of exercise can elicit substantial changes in the immune system. ${ }^{29}$ Described as the "acutestress induced immunoenhancement hypothesis", the increases in epinephrine, cortisol, heart rate and blood pressure encompass the acute response to exercise. ${ }^{30}$ Alongside these physiological adjustments is the well-stablished leukocytosis response, the transient increase in muscle-secreted inflammatory cytokines, and the exercise-induced muscle damage leading to leukocyte trafficking to the tissue. These orchestrated adjustments have been postulated to stimulate the activation of immune surveillance in anticipation of antigen entry, ${ }^{28,31}$ which may be of particular relevance to vaccination. ${ }^{30}$ Although the clinical benefit of physical activity on vaccines efficacy is commonly inferred from the quantified antibody, neutralization activity or cell-mediated responses, this postulation finds support in a populationbased cohort study, in which moderately- and highly-active individuals were less likely to experience an influenza-coded visit to a physician or emergency department. ${ }^{32}$ Whether SARS-CoV-2 vaccine efficacy may be modulated by physical activity and how it occurs remain to be investigated.

Our data is strengthened by the large prospective cohort of immunocompromised patients with ARD, the assessment of immunogenicity using both SARS-CoV-2 IgG and NAb, and the robust control for numerous covariates. Limitations include the use of questionnaire to assess physical activity, which is prone to recall bias and overreporting; lack of estimates of vaccine effectiveness to bridge to the immunogenicity data; short-term assessment of immunogenicity, precluding any firm conclusions on the persistency of the observed responses; lack of assessment of cell-mediated immune responses; observational nature of the study, hampering causative inferences; and the constraint of the results to the vaccine tested in this study. In this regard, CoronaVac seems to evoke less protective titer compared to others, a response associated with lower protection from SARS-CoV-2 infection. ${ }^{33}$ This underpins the clinical relevance of the current findings; conversely, it is uncertain whether physical activity may also associate with enhanced responses to other vaccine platforms able to elicit higher protective titer, as a ceiling effect may exist at least for heathy individuals.

Cumulative evidence has shown that physical activity is associated with better outcomes in COVID-19 patients. Consistently active individuals seem less susceptible to COVID-19-related ICU admission and mortalitv 34 Thic ctudv hrince novel evidence suggesting that a physical active lifestyle may also boost Loading [MathJax]/jax/output/CommonHTML/jax.js 
SARS-CoV-2 vaccine immunogenicity, a finding of particular relevance for people with dysfunctional immune system. Collectively, these data reinforce the need for a global call for action to delivery physical activity during the COVID-19 pandemic, with particular emphasis to groups with reduced immune function. Randomized controlled trials are necessary to confirm the efficacy of physical activity in enhancing vaccine responses, and to stablish the optimal dose to elicit the greatest benefits.

\section{Methods}

\section{Ethics statement}

The protocol was approved by the National and Institutional Ethical Committee of the Hospital das Clínicas. Written informed consent was obtained from each participant before enrollment.

\section{Study design and setting}

This was a prospective cohort study within the protocol of an open-label, single-arm, phase 4 vaccination trial (clinicaltrials.gov \#NCT04754698), conducted at a tertiary referral hospital in Sao Paulo, Brazil.

\section{Participants}

ARD patients aged $\geq 18$ years and diagnosed with rheumatoid arthritis, systemic lupus erythematosus, axial spondyloarthritis, psoriatic arthritis, primary vasculitis, primary Sjögren's syndrome, systemic sclerosis, systemic autoimmune myopathies and primary antiphospholipid syndrome, following previously reported criteria. ${ }^{9}$ Additionally, a group of individuals without ARD, HIV or other conditions requiring immunosuppressive therapy were also studied. Exclusion criteria were: history of anaphylactic response to vaccine components, acute febrile illness or symptoms compatible to COVID-19 at vaccination, Guillain-Barré syndrome, decompensated heart failure (class III or IV), demyelinating disease, previous vaccination with any SARS-CoV-2 vaccine, history of live virus vaccine up to four weeks before, inactivated virus vaccine up to two weeks before, and receipt of blood products up to six months before the study, hospitalized patients, and pre-vaccination COVID-19 assessed by anti-SARS-CoV-2 S1/S2 IgG and/or neutralizing antibodies (NAb). Participants who had RT-PCR-confirmed COVID-19 after receiving 1 st vaccine dose were excluded. ${ }^{9}$

\section{Vaccination}

Participants underwent a two-dose schedule of CoronaVac (Sinovac Life Sciences, Beijing, China, batch \#20200412) as previously described. ${ }^{9}$ The 1 st dose was administered on February 9-10, 2021 (D0) and the 2nd dose was given on March 9-10, 2021 (D28). Blood samples (20mL) from all participants were obtained at D0, D28, and D69 (six weeks after 2nd dose) at the Hospital Convention Center. Sera were stored in a $-70^{\circ} \mathrm{C}$ freezer for posterior analysis.

\section{Physical activity level and sedentary behavior}


Physical activity and sedentary behavior were assessed by experienced researchers through telephone survey. Physical activity level survey comprised eight questions addressing four different physical activity domains: leisure-time, household activities, work, and commuting (Supplementary Material 1).

Participants were asked how many days/week and minutes/day were spent in moderate-to-vigorous intensity activities in each domain, and summed for total time spent in moderate-to-vigorous physical activity. Participants were classified as physically active or inactive according to WHO Guidelines (i.e., physical inactivity defined as $<150 \mathrm{~min} /$ week of moderate-to-vigorous intensity aerobic activity). ${ }^{35}$

Sedentary behavior was assessed by asking participants how many hours/day were spent sitting throughout the week and weekend days. Sedentary status (yes: $\geq 8$ hours/day; or no: $<8$ hours/day) ${ }^{26}$ was used in combination with physical activity to test whether these would additively influence the outcomes.

Six telephone calls and text messages were made to each participant before deeming the individual as a non-respondent.

\section{Immunogenicity}

Immunogenicity was assessed at D69 using seroconversion rates of total anti-SARS-CoV-2 S1/S2 IgG (SC), geometric mean titers of anti-S1/S2 IgG (GMT) and their factor-increase in GMT (FI-GMT), frequency of NAb and median (interquartile range) of neutralizing activity.

\section{Anti-SARS-CoV-2 S1/S2 IgG antibodies}

Human IgG antibodies against S1 and S2 proteins in RBD (Indirect ELISA, LIAISON® SARS-CoV-2 S1/S2 IgG, DiaSorin, Italy) were assessed by chemiluminescent immunoassay. SC was defined as positive serology (>15.0 UA/mL) post vaccination (considering all participants were negative for pre-vaccination serology at baseline). GMT was calculated attributing $1.9 \mathrm{UA} / \mathrm{mL}$ (half of the lower limit of quantification) to undetectable levels ( $<3.8 \mathrm{UA} / \mathrm{mL}$ ). FI-GMT was determined as the ratio between GMT after and before vaccination and are presented as geometric means and $95 \%$ confidence intervals (Cls).

\section{SARS-CoV-2 cPass virus-NAb}

Circulating NAb against SARS-CoV-2 was assessed using the SARS-CoV-2 sVNT Kit (GenScript, Piscataway, NJ, USA), which detects neutralizing antibodies that block the interaction between RBD in the viral spike glycoprotein with angiotensin-converting enzyme 2 (ACE2) cell surface receptor. Tests were performed on ETI-MAX-3000 (DiaSorin, Italy). Samples were classified as either "positive" or "negative" (inhibition $\geq 30$ or $<30 \%$, respectively), as suggested by the manufacturer. Median (interquartile range) of the percentage of neutralizing activity was calculated for positive samples.

\section{Statistical analysis}

Baseline characteristics and outcomes for both ARD patients and non-ARD individuals measured after vaccination were compared across activity levels using $\chi^{2}$ test for categorical variables, exact test for Loading [MathJax]/jax/output/CommonHTML/jax.js e Kruskal-Wallis test for continuous variables. Model-based 
analyses were then performed controlling for age ( $<60$ or $\geq 60$ years), sex, and BMI ( $<25 \mathrm{~kg} / \mathrm{m}^{2} ; 25-30$ $\mathrm{kg} / \mathrm{m}^{2} ;>30 \mathrm{~kg} / \mathrm{m}^{2}$ ). For ARD patients, further controls included use of prednisone, immunosuppressants and biologics. Confounders were selected based on a Direct Acyclic Graph (DAG; www.dagitty.net) (Supplementary Fig. 1). ${ }^{36}$ DAG was developed from a priori knowledge to identify a minimum, but sufficient set of covariates to remove confounding from statistical analysis. ${ }^{37}$ Data following vaccination and activity status were added as fixed effects and we conducted logistic regression to estimate odds ratios (ORs) and $95 \% \mathrm{Cls}$ with binary data obtained for frequency of IgG SC and NAb positivity. We conducted Tobit regression to account for floor effects and frequency minimum values obtained for neutralizing activity and natural log transformed IgG and FI-GMT. Tobit regression coefficients and 95\%Cls for log transformed dependent variables were back transformed and presented as percent changes. An exploratory analysis clustering physical activity and sedentary status (active/sedentary; inactive/non-sedentary; active/non-sedentary; inactive/sedentary) was conducted for ARD patients. A further exploratory analysis tested a possible dose-response between total weekly volume of physical activity $(0-30 ; 31-149 ; 150-349 ; \geq 350 \mathrm{~min})$ and immunogenicity data. Analyses were conducted using R-statistical environment (R-4.1.0 for Windows).

\section{Declarations}

\section{Acknowledgments}

The authors are thankful to Marta H. Lopes, Tatiana Pedrosa, Antonio José Rodrigues Pereira, Solange Fusco, Priscila Tagliaferro Rojo, and Central Laboratory Division of the Clinical Hospital of the School of Medicine (University of Sao Paulo) for the technical assistance.

\section{Author Contributions}

N.E.A., S.G.P., A.C.M.R., C.G.S.S., E.F.N.Y, C.A.S. and E.B., conceived and designed the study, and supervised clinical data management. B.G., I.R.L., R.P.S., A.J.P., B.C.M., F.I.S., S.M.S., N.E.A., S.G.P., A.C.M.R., C.G.S.S., E.F.N.Y, C.A.S., H.R. and E.B participated in data collection. B.G., I.R.L., A.J.P., P.S., P.C.H. and H.R. analyzed and interpreted the data. B.G., H.R., and E.B. wrote the manuscript with inputs from all authors. All authors read and approved the final version.

\section{Competing Interest Statement}

The authors have no conflict of interests. 
This trial is sponsored by grants from Fundação de Amparo à Pesquisa do Estado de São Paulo (FAPESP) (\#2015/03756-4 to NEA, SGP, CAS and EB; \#2015/26937-4 to AJP; \#2017/13552-2 to BG; \#2020/04877-8 to IRL; \#2017/23688-9 to RPS; \#2019/14820-6 to BCM; \#2019/14819-8 to FIS; \#2019/15231-4 to SMS), Conselho Nacional de Desenvolvimento Científico e Tecnológico (CNPq) (\#305242/2019-9 to EB, \#304984/2020-5 to CAS) and B3 - Bolsa de Valores do Brasil. Instituto Butantan supplied the study's product and had no other role in the trial.

\section{Data availability}

All background information on non-ARD individuals and clinical information for ARD patients in this study are available from corresponding author on reasonable request.

\section{References}

1. Haas, E. J. et al. Impact and effectiveness of mRNA BNT162b2 vaccine against SARS-CoV-2 infections and COVID-19 cases, hospitalisations, and deaths following a nationwide vaccination campaign in Israel: an observational study using national surveillance data. Lancet (London, England) 397, 1819-1829 (2021).

2. Hall, V. J. et al. COVID-19 vaccine coverage in health-care workers in England and effectiveness of BNT162b2 mRNA vaccine against infection (SIREN): a prospective, multicentre, cohort study. Lancet 397, 1725-1735 (2021).

3. Vasileiou, E. et al. Interim findings from first-dose mass COVID-19 vaccination roll-out and COVID-19 hospital admissions in Scotland: a national prospective cohort study. Lancet 397, 1646-1657 (2021).

4. Geisen, U. M. et al. Immunogenicity and safety of anti-SARS-CoV-2 mRNA vaccines in patients with chronic inflammatory conditions and immunosuppressive therapy in a monocentric cohort. Ann. Rheum. Dis. (2021) doi:10.1136/annrheumdis-2021-220272.

5. Boyarsky, B. J. et al. Immunogenicity of a Single Dose of SARS-CoV-2 Messenger RNA Vaccine in Solid Organ Transplant Recipients. JAMA 325, 1784-1786 (2021).

6. Boyarsky, B. J. et al. Antibody Response to 2-Dose SARS-CoV-2 mRNA Vaccine Series in Solid Organ Transplant Recipients. JAMA 325, 2204-2206 (2021).

7. Simon, D. et al. SARS-CoV-2 vaccination responses in untreated, conventionally treated and anticytokine-treated patients with immune-mediated inflammatory diseases. Ann. Rheum. Dis. (2021) doi:10.1136/annrheumdis-2021-220461.

8. Boyarsky, B. J. et al. Antibody response to a single dose of SARS-CoV-2 mRNA vaccine in patients with rheumatic and musculoskeletal diseases. Annals of the rheumatic diseases (2021) doi:10.1136/annrheumdis-2021-220289. 
9. Medeiros-Ribeiro, A. C. et al. Immunogenicity and safety of the CoronaVac inactivated vaccine in patients with autoimmune rheumatic diseases: a phase 4 trial. Nat. Med. (2021) doi:10.1038/s41591-021-01469-5.

10. Simpson, R. J., Kunz, H., Agha, N. \& Graff, R. Exercise and the Regulation of Immune Functions. Prog. Mol. Biol. Transl. Sci. 135, 355-380 (2015).

11. Chastin, S. F. M. et al. Effects of Regular Physical Activity on the Immune System, Vaccination and Risk of Community-Acquired Infectious Disease in the General Population: Systematic Review and Meta-Analysis. Sports medicine (Auckland, N.Z.) 1-14 (2021) doi:10.1007/s40279-021-01466-1.

12. Nicholson, K. G., Wood, J. M. \& Zambon, M. Influenza. Lancet 362, 1733-1745 (2003).

13. Pascoe, A. R., Fiatarone Singh, M. A. \& Edwards, K. M. The effects of exercise on vaccination responses: a review of chronic and acute exercise interventions in humans. Brain. Behav. Immun. 39, 33-41 (2014).

14. Hilleman, M. R. Vaccines in historic evolution and perspective: a narrative of vaccine discoveries. Vaccine 18, 1436-1447 (2000).

15. Villari, P., Manzoli, L. \& Boccia, A. Methodological quality of studies and patient age as major sources of variation in efficacy estimates of influenza vaccination in healthy adults: a meta-analysis. Vaccine 22, 3475-3486 (2004).

16. Polack, F. P. et al. Safety and Efficacy of the BNT162b2 mRNA Covid-19 Vaccine. N. Engl. J. Med. 383, 2603-2615 (2020).

17. World Health Organization, Strategic Advisory Group of Experts (SAGE) on Immunization \& SAGE Working Group on COVID-19 Vaccines. Interim recommendations for use of the inactivated COVID-19 vaccine, CoronaVac, developed by Sinovac.

18. Grant, R. W. et al. Cardiovascular exercise intervention improves the primary antibody response to keyhole limpet hemocyanin (KLH) in previously sedentary older adults. Brain. Behav. Immun. 22, 923-932 (2008).

19. Keylock, K. T. et al. Higher antibody, but not cell-mediated, responses to vaccination in high physically fit elderly. J. Appl. Physiol. 102, 1090-1098 (2007).

20. Kohut, M. L., Cooper, M. M., Nickolaus, M. S., Russell, D. R. \& Cunnick, J. E. Exercise and psychosocial factors modulate immunity to influenza vaccine in elderly individuals. J. Gerontol. A. Biol. Sci. Med. Sci. 57, M557-62 (2002).

21. Kohut, M. L. et al. Moderate exercise improves antibody response to influenza immunization in older adults. Vaccine 22, 2298-2306 (2004).

22. Schuler, P. B., Leblanc, P. A. \& Marzilli, T. S. Effect of physical activity on the production of specific antibody in response to the 1998-99 influenza virus vaccine in older adults. J. Sports Med. Phys. Fitness 43, 404 (2003).

23. Smith, T. P., Kennedy, S. L. \& Fleshner, M. Influence of age and physical activity on the primary in vivo antibody and T cell-mediated responses in men. J. Appl. Physiol. 97, 491-498 (2004). 
24. Woods, J. A. et al. Cardiovascular Exercise Training Extends Influenza Vaccine Seroprotection in Sedentary Older Adults: The Immune Function Intervention Trial. J. Am. Geriatr. Soc. 57, 2183-2191 (2009).

25. Yang, Y. et al. Effects of a Taiji and Qigong intervention on the antibody response to influenza vaccine in older adults. Am. J. Chin. Med. 35, 597-607 (2007).

26. Ekelund, U. et al. Does physical activity attenuate, or even eliminate, the detrimental association of sitting time with mortality? A harmonised meta-analysis of data from more than 1 million men and women. Lancet 388, 1302-1310 (2016).

27. Stamatakis, E. et al. Sitting Time, Physical Activity, and Risk of Mortality in Adults. J. Am. Coll. Cardiol. 73, 2062-2072 (2019).

28. Edwards, K. M. \& Booy, R. Effects of exercise on vaccine-induced immune responses. Hum. Vaccin. Immunother. 9, 907-910 (2013).

29. Edwards, K. M. et al. Acute exercise enhancement of pneumococcal vaccination response: A randomised controlled trial of weaker and stronger immune response. Vaccine 30, 6389-6395 (2012).

30. Edwards, K. M., Burns, V. E., Carroll, D., Drayson, M. \& Ring, C. The acute stress-induced immunoenhancement hypothesis. Exerc. Sport Sci. Rev. 35, 150-155 (2007).

31. Viswanathan, K., Daugherty, C. \& Dhabhar, F. S. Stress as an endogenous adjuvant: augmentation of the immunization phase of cell-mediated immunity. Int. Immunol. 17, 1059-1069 (2005).

32. Siu, E., Campitelli, M. A. \& Kwong, J. C. Physical activity and influenza-coded outpatient visits, a population-based cohort study. PLoS One 7, e39518 (2012).

33. Khoury, D. S. et al. Neutralizing antibody levels are highly predictive of immune protection from symptomatic SARS-CoV-2 infection. Nat. Med. (2021) doi:10.1038/s41591-021-01377-8.

34. Sallis, R. et al. Physical inactivity is associated with a higher risk for severe COVID-19 outcomes: a study in 48440 adult patients. Br. J. Sports Med. (2021) doi:10.1136/bjsports-2021-104080.

35. Bull, F. C. et al. World Health Organization 2020 guidelines on physical activity and sedentary behaviour. Br. J. Sports Med. 54, 1451-1462 (2020).

36. Joffe, M., Gambhir, M., Chadeau-Hyam, M. \& Vineis, P. Causal diagrams in systems epidemiology. Emerg. Themes Epidemiol. 9, 1 (2012).

37. Robins, J. M. Data, design, and background knowledge in etiologic inference. Epidemiology 12, 313320 (2001).

\section{Tables}




\begin{tabular}{|c|c|}
\hline & $\begin{array}{r}\text { ARD } \\
(n=898)\end{array}$ \\
\hline ears & $52.0[41.0-62.0]$ \\
\hline male & $683(76.1)$ \\
\hline $\mathrm{t}, \mathrm{kg}$ & $71.4[60.3-82.4]$ \\
\hline t, $\mathrm{cm}$ & $160.0[155.0-166.0]$ \\
\hline$g / m^{2}$ & $27.5[24.2-31.2]$ \\
\hline eight/obese & $567(63.2)$ \\
\hline sian race & $488(54.3)$ \\
\hline $\mathrm{ng}$ & $78(8.7)$ \\
\hline \multicolumn{2}{|l|}{ bidities } \\
\hline temic arterial hypertension & $408(45.4)$ \\
\hline betes mellitus & $105(11.7)$ \\
\hline lipidemia & $247(27.5)$ \\
\hline diomyopathy & $54(6.0)$ \\
\hline onic renal disease & $44(4.9)$ \\
\hline onic obstructive pulmonary disease & $15(1.7)$ \\
\hline ima & $38(4.2)$ \\
\hline rstitial lung disease & $73(8.1)$ \\
\hline nonary hypertension & $11(1.2)$ \\
\hline natologic disease & $3(0.3)$ \\
\hline atic disease & $36(4.0)$ \\
\hline cer & $9(1.0)$ \\
\hline $\mathrm{ke}$ & $28(3.1)$ \\
\hline erculosis & $2(0.2)$ \\
\hline
\end{tabular}

onic inflammatory arthritis (RA, axSpA, PsA)

it therapy

dnisone

ogic

e presented as median [interquartile range] and $n$ (\%). ARD, autoimmune rheumatic disease; BMI, ass index; RA, rheumatoid arthritis; axSpA, axial spondyloarthritis; PsA, psoriatic arthritis; SLE, c lupus erythematosus; SSc, systemic sclerosis; pSSj, primary Sjögren syndrome; IIMM, idiopathic ıatory myopathies; PAPS, primary antiphospholipid syndrome. Biologics include TNF inhibitor, !pt, tocilizumab, belimumab, secukinumab, rituximab, ustekinumab. Immunosuppressants include exate, leflunomide, mycophenolate mofetil, azathioprine, tofacitinib, cyclophosphamide, tacrolimus and orine.

lissing data for weight and BMI $(n=1)$. 
able 2. Characteristics of autoimmune rheumatic diseases (ARD) patients and non-ARD ıdividuals according to physical activity status.

\begin{tabular}{lrrrrrr}
\hline & $\begin{array}{r}\text { ARD Active } \\
(n=494)\end{array}$ & $\begin{array}{r}\text { ARD } \\
\text { Inactive } \\
(n=404)\end{array}$ & $\begin{array}{r}P- \\
\text { value }\end{array}$ & $\begin{array}{r}\text { Non-ARD } \\
\text { Active } \\
(n=128)\end{array}$ & $\begin{array}{r}\text { Non-ARD } \\
\text { Inactive } \\
(n=69)\end{array}$ & $\begin{array}{r}P \text { - } \\
\text { value }\end{array}$ \\
\hline ge, years & $47.0[39.0-$ & $56.0[45.0-$ & $<.001$ & $48.0[34.0-$ & $47.0[38.0-$ & .993 \\
& $58.0]$ & $66.0]$ & & $59.0]$ & $55.0]$ & \\
ge, $<60$ years & $389(78.7)$ & $236(58.4)$ & $<.001$ & $100(78.1)$ & $56(81)$ & .617 \\
ex, female & $378(76.5)$ & $305(75.5)$ & .780 & $84(65.6)$ & $47(68)$ & .845 \\
MI, $\mathrm{kg} / \mathrm{m}^{2}$ & $27.5[24.2-$ & $27.5[24.2-$ & .343 & $26.4[23.4-$ & $26.8[24.8-$ & .397 \\
rednisone & $31.6]$ & $31.6]$ & & $30.2]$ & $31.0]$ & \\
iologic & $186(37.7)$ & $170(42.1)$ & $<.001$ & - & - & - \\
nmunosuppressants & $164(33.2)$ & $163(40.3)$ & .032 & - & - & - \\
\hline
\end{tabular}

əsults are expressed in median [interquartile range] and $n$ (\%). BMI, body mass index; ARD, autoimmune teumatic diseases.

ote: Missing data for BMI (ARD, $n=1$; non-ARD, $n=6$ ).

\section{Figures}



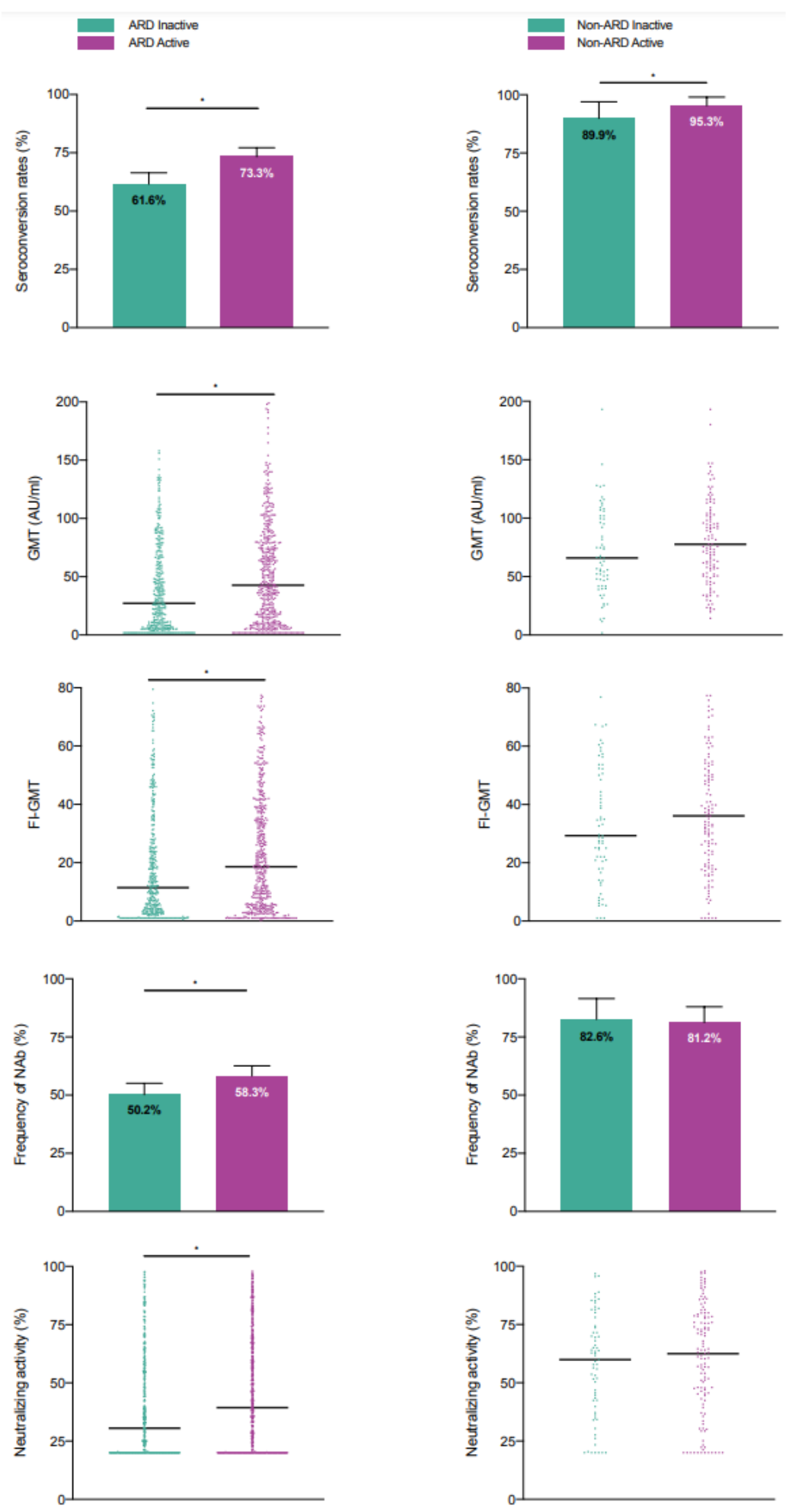

\section{Figure 1}

Unadjusted analysis for immunogenicity data in autoimmune rheumatic diseases patients (ARD) (left) and in non-ARD individuals (right). ${ }^{*} \mathrm{P}<.05$. Seroconversion was defined as a positive serology (IgG titer $>$ $15 \mathrm{AU} / \mathrm{ml}$ ) for anti-SARS-CoV-2 S1/S2 IgG antibodies after vaccination (Indirect ELISA, LIAISON® SARSCoV-2 S1/S2 IgG, DiaSorin, Italy). Positivity for NAb was defined as a neutralizing activity $\geq 30 \%$ (cPass 
sVNT Kit, GenScript, Piscataway, USA). Data are expressed as median and Cl 95\% for frequency of SC and NAb positivity, and mean and individual data for neutralizing activity, GMT and FI-GMT.

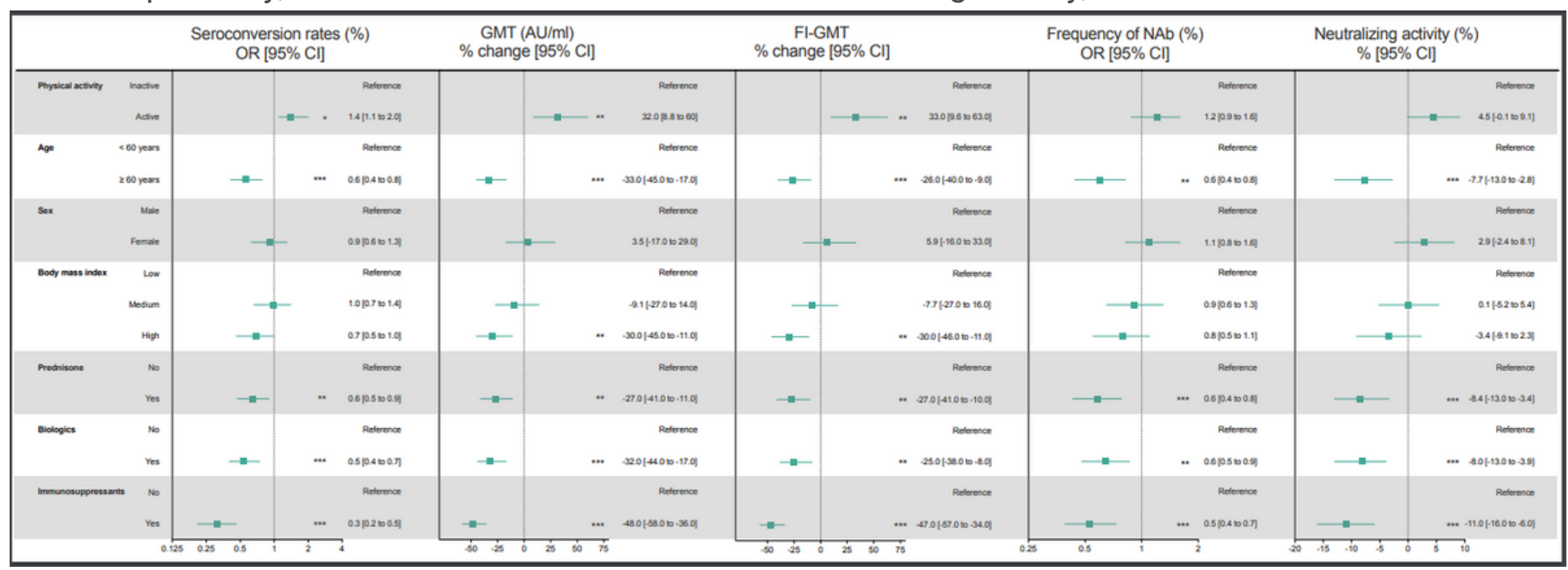

Figure 2

Adjusted risk factors for immunogenicity data in autoimmune rheumatic diseases (ARD) patients. Logistic regression to estimate odds ratios (ORs) and 95\% confidence intervals (Cls) with binary data obtained for frequency of seroconversion rates of total anti-SARS-Cov-2 S1/S2 IgG (SC) and neutralizing antibodies (NAb) positivity. Tobit regression was used for natural log transformed GMT, FI-GMT and neutralizing activity. Data expressed as either percent or percent change $[95 \% \mathrm{Cl}])$ in patients with autoimmune rheumatic diseases following a vaccine against SARS-CoV-2. ${ }^{*} P<.05,{ }^{*} \mathrm{P}<.01,{ }^{\star} * \star \mathrm{P}<.001$. Seroconversion was defined as a positive serology (IgG titer $\geq 15 \mathrm{AU} / \mathrm{ml}$ ) for anti-SARS-CoV-2 S1/S2 IgG antibodies after vaccination (Indirect ELISA, LIAISON® SARS-CoV-2 S1/S2 IgG, DiaSorin, Italy). Positivity for NAb was defined as a neutralizing activity $\geq 30 \%$ (cPass sVNT Kit, GenScript, Piscataway, USA).

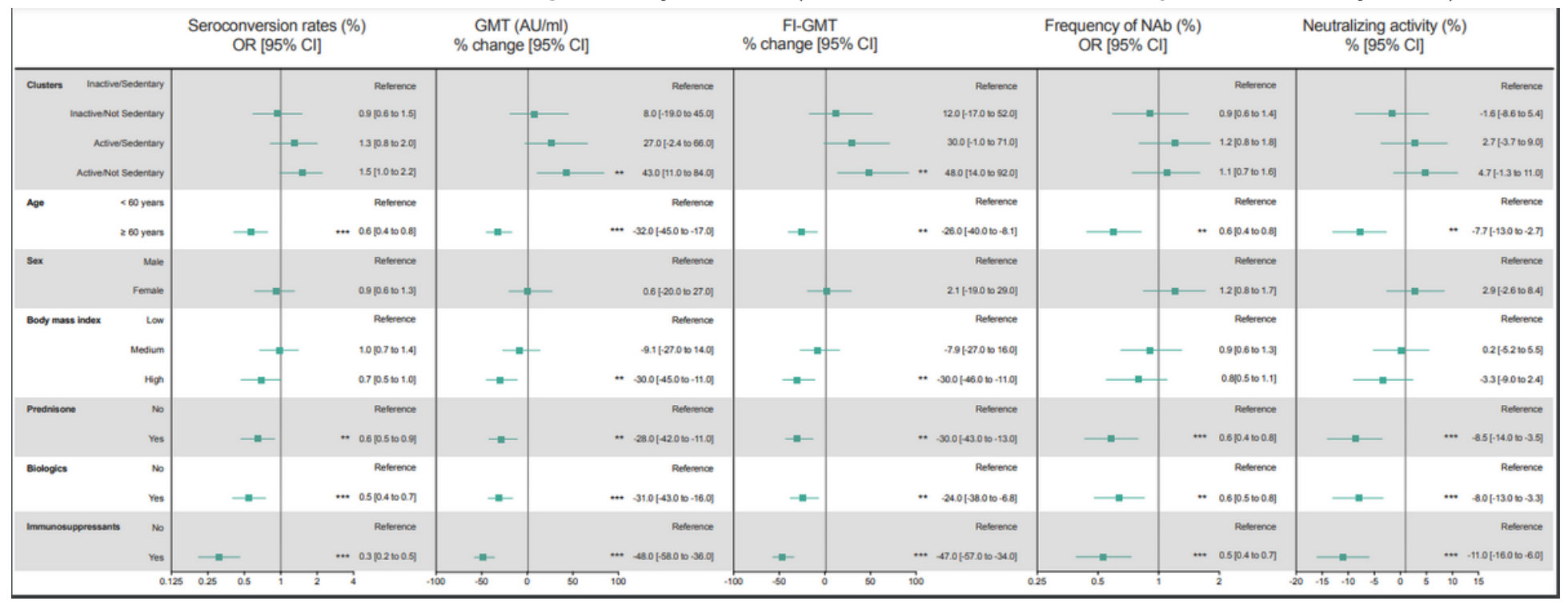

Figure 3 
Adjusted risk factors for immunogenicity data in autoimmune rheumatic diseases (ARD) patients clustered for physical activity and sedentary behavior. Logistic regression to estimate odds ratios (ORs) and $95 \%$ confidence intervals (Cls) with binary data obtained for frequency of seroconversion rates of total anti-SARS-Cov-2 S1/S2 IgG (SC) and neutralizing antibodies (NAb) positivity. Tobit regression was used for natural log transformed GMT, FI-GMT and neutralizing activity. Data expressed as either percent or percent change $[95 \% \mathrm{Cl}]$ ) in patients with autoimmune rheumatic diseases following a vaccine against SARS-CoV-2. ${ }^{*} P<.05,{ }^{*} \mathrm{P}<.01,{ }^{*} * * \mathrm{P}<.001$. Seroconversion was defined as a positive serology (IgG titer $\geq$ $15 \mathrm{AU} / \mathrm{ml}$ ) for anti-SARS-CoV-2 S1/S2 IgG antibodies after vaccination (Indirect ELISA, LIAISON® SARSCoV-2 S1/S2 IgG, DiaSorin, Italy). Positivity for NAb was defined as a neutralizing activity $\geq 30 \%$ (cPass sVNT Kit, GenScript, Piscataway, USA).

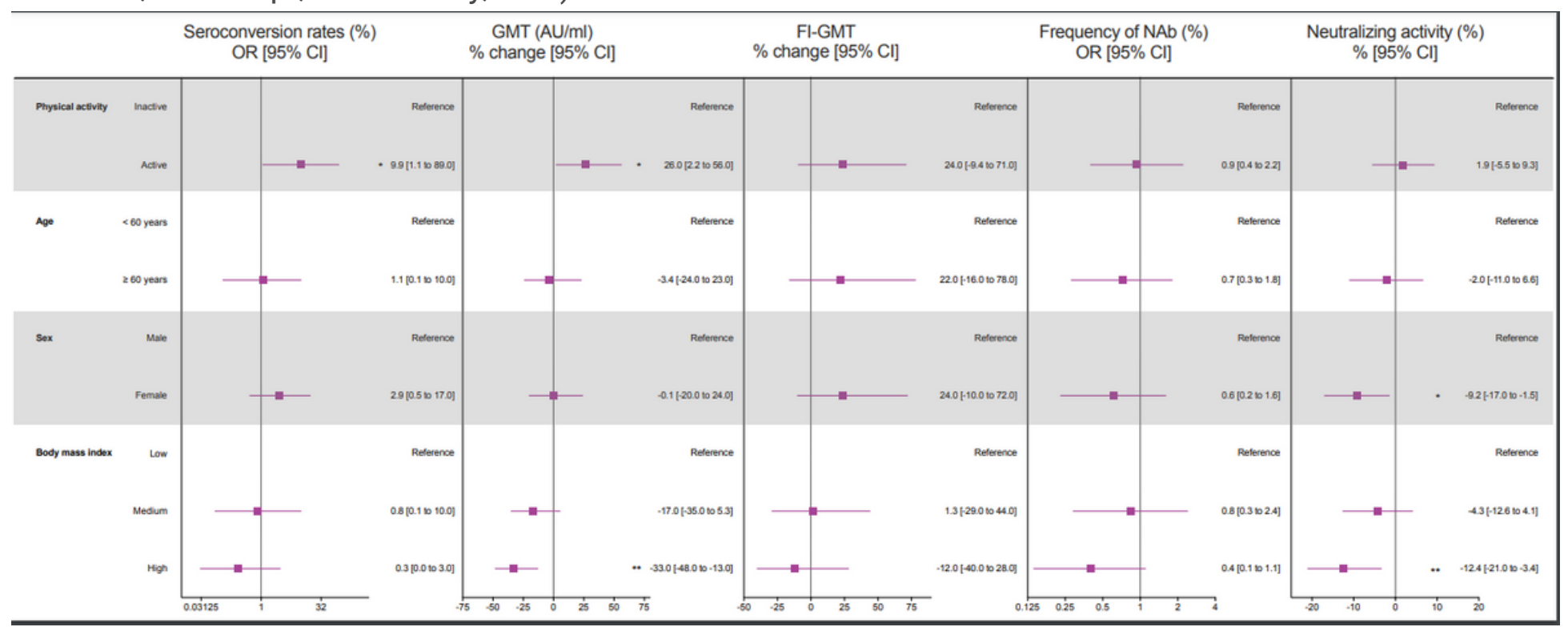

\section{Figure 4}

Adjusted risk factors for immunogenicity data in non-autoimmune rheumatic diseases (non-ARD) individuals. Logistic regression to estimate odds ratios (ORs) and 95\% confidence intervals (Cls) with binary data was used for frequency of seroconversion rates of total anti-SARS-Cov-2 S1/S2 IgG (SC) and neutralizing antibodies (NAb) positivity. Tobit regression was used for natural log transformed GMT, FIGMT and neutralizing activity. ${ }^{*} \mathrm{P}<.05,{ }^{* \star} \mathrm{P}<.01,{ }^{\star \star *} \mathrm{P}<.001$. Seroconversion was defined as a positive serology (IgG titer $\geq 15 \mathrm{AU} / \mathrm{ml}$ ) for anti-SARS-CoV-2 S1/S2 lgG antibodies after vaccination (Indirect ELISA, LIAISON® SARS-CoV-2 S1/S2 IgG, DiaSorin, Italy). Positivity for NAb was defined as a neutralizing activity $\geq 30 \%$ (cPass sVNT Kit, GenScript, Piscataway, USA).

\section{Supplementary Files}

This is a list of supplementary files associated with this preprint. Click to download.

- Supplementaryinformation.pdf 